\title{
Supervised Principal Geodesic Analysis on Facial Surface Normals for Gender Classification
}

\author{
Jing Wu, William A.P. Smith, and Edwin R. Hancock \\ Department of Computer Science, The University of York, York, YO10 5DD, UK \\ $\{$ jwu, wsmith, erh\}@cs.york.ac.uk
}

\begin{abstract}
In this paper, we perform gender classification based on facial surface normals (facial needle-maps). We improve our previous work in [6] by using a non-Lambertian Shape-from-Shading (SFS) method to recover the surface normals, and develop a novel supervised principal geodesic analysis (PGA) to parameterize the facial needle-maps. Experimental results demonstrate the feasibility of gender classification based on facial needle-maps, and shows that incorporating pairwise relationships between the labeled data improves the gender discriminating powers in the leading PGA eigenvectors and gender classification accuracy.
\end{abstract}

\section{Introduction}

Although the determination of gender from facial images has been the focus of sustained activity in both computer vision [1, 2, 3] and psychology [4, 5], most of the existing work is based on 2D images. Although it has been shown that gender is revealed by both the $2 \mathrm{D}$ texture and the $3 \mathrm{D}$ shape of the human face [4, few studies have investigated the role of 3D shape in gender classification 2. To some extent this is due to the more complex computations required in 3D face shape analysis, and the limited effectiveness and high cost of the current 3D sensors. In [6], we have investigated the feasibility of gender classification based on 2.5D facial surface normals (or facial needle-maps), which exhibits the shape information from a fixed viewpoint and can be recovered from single images using shape-from-shading (SFS). In [6], an iterative principal geodesic SFS method, which combines the facial texture and shape information implicitly, is applied to facial images. However, this SFS method is only suitable for surfaces which exhibit Lambertian reflectance. Human face surfaces, on the other hand, do not strictly obey Lambert's law due to specularities caused by perspiration etc. In this paper, we apply an improved SFS method [7], which is suitable to non-Lambertian surfaces, to facial images. This method satisfies the brightness constraint using the Torrance and Sparrow reflectance model [8], and guarantees the accuracy of the recovered needle-maps by using a statistical height model.

Another improvement to [6] is the parameterisation of the needle-maps. Facial needle-maps can be considered as points lying on the $S^{2}(N)$ manifold. To parameterize data lying on this Riemmanian manifold, in [6], we use principal geodesic analysis (PGA) 9], 10. However PGA locates the projections that maximize the 
variance of the data. Usually the projections that maximize the variance are not those that separate the data into distinct clusters. As a result the PGA parameters do not usually reveal cluster structure. In [11, the authors view PCA as locating projection that maximizes the sum of squared pairwise distances between the projected data. By associating a dissimilarity weight with each pairwise distance, they obtain a supervised PCA projection that emphasizes the inter-cluster separation. In this paper, we develop a novel supervised variant of PGA by extending this idea to the $S^{2}(N)$ space for the analysis of surface normals. By incorporating pairwise gender relationships, supervised PGA makes use of the interclass structure, and improves the gender discriminating power of the leading principal geodesics. We also show that the supervised PGA method can be viewed as applying standard PGA on the weighted facial needle-maps with the applied weight matrices specific to each facial needle-map. This links supervised PGA with the weighted PGA, we proposed before [12. However the weight matrix is weighted PGA is a common one which can be obtained from a priori knowledge.

The outline of the paper is as follows. Section 2 reviews the PGA technique, and gives a detailed description of supervised PGA. Section 3 describes the non-Lambertian SFS method used to generate the needle-maps. Section 4 describes the classifier used for gender classification. Experiments on ground-truth needle-maps and brightness images are presented in Section 5. Finally, Section 6 concludes the paper and offers directions for future investigation.

\section{Supervised Principal Geodesic Analysis}

\subsection{Principal Geodesic Analysis}

A surface normal $n$ may be considered as a point residing on a spherical manifold $n \in S^{2}$. Facial needle-maps, which are fields of $N$ surface normals, may be considered as a point on the manifold $S^{2}(N)=\prod_{i=1}^{N} S^{2}$. For the analysis of data on this manifold, we turn to the intrinsic mean and PGA 9, [10.

Given $u \in T_{n} S^{2}$, a non-zero vector on the tangent plane to $S^{2}$, the exponential map, denoted $\operatorname{Exp}_{n}$, maps $u$ to the point $\operatorname{Exp}_{n}(u)$ along the geodesic in the direction of $u$ at distance $\|u\|$ from $n$. This is illustrated in Fig. 1 . The $\log$ map, denoted $\log _{n}$ is the inverse of the exponential map. In $S^{2}(N)$ space, the exponential and log maps are the products of $N$ copies of the maps for $S^{2}$.

Intrinsic means minimize the sum-of-squared Riemannian distances on $M$ : $\mu=\arg \min _{x \in M} \sum_{i=1}^{K} d\left(x, x_{i}\right)^{2}$. For a spherical manifold, the intrinsic mean of a set of surface normals $n_{1}, \ldots, n_{K} \in S^{2}$ can be found using the gradient descent method of Pennec [10]: $\mu^{(t+1)}=\operatorname{Exp}_{\mu^{(t)}}\left(\frac{1}{K} \sum_{i=1}^{K} \log _{\mu^{(t)}}\left(n_{i}\right)\right)$.

For data on a connected, complete manifold, PGA finds a sequence of nested geodesics that maximize the projected variance. In the spherical case, a geodesic corresponds to a great circle. The projection $\pi_{G}: S^{2} \rightarrow G$ is defined as: $\pi_{G}\left(n_{1}\right)=$ $\arg \min _{n \in G} d\left(n_{1}, n\right)^{2}$. For a geodesic $G$ passing through the intrinsic mean $\mu$, this projection can be approximated linearly in the tangent space $T_{\mu} S^{2}$ : 


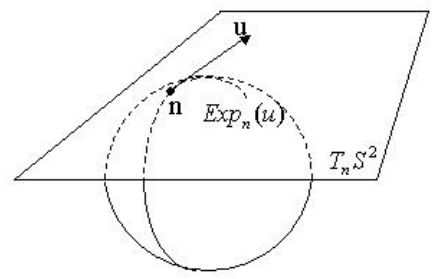

Fig. 1. The exponential map

$$
\log _{\mu}\left(\pi_{G}\left(n_{1}\right)\right) \approx \sum_{i=1}^{m} v_{i} \cdot \log _{\mu}\left(n_{1}\right)
$$

where $v_{1}, \ldots v_{d}$ is an orthonormal basis for $T_{\mu} S^{2}$ obtained through standard PCA.

\subsection{Supervised Principal Geodesic Analysis}

Supervised PCA is developed by Koren and Carmel [1]. They view PCA as computing the p-dimensional projection that maximizes $\sum_{i<j}\left(d_{i s t}^{p}\right)_{i j}^{2}$, where $d i s t_{i j}^{p}$ is the Euclidean distance between the projected data $i$ and $j$. By incorporating a symmetric and nonnegative pairwise weight matrix $\left\{d_{i j}\right\}_{i, j=1}^{n}$, where $d_{i j}$ is the dissimilarity of data $i$ and $j$, they generalized PCA to seek the projection that maximizes the sum of weighted squared pairwise distances

$$
d i s t_{\text {sum }}^{p}=\sum_{i<j} d_{i j}\left(d i s t_{i j}^{p}\right)^{2} .
$$

Koren and Carmel [11] proved that the p-dimensional projection that maximizes (2) is obtained by taking the $p$ principal eigenvectors of the matrix $X^{T} L X$, where $L$ is the Laplacian associated with the dissimilarities, and $X$ is the row-wise data matrix. For labeled data, this weighted PCA turns to be supervised PCA when underweights the intra-cluster pairwise dissimilarities:

$$
d_{i j}^{l a b e l e d}= \begin{cases}t \cdot d_{i j}, & \mathrm{i} \text { and } \mathrm{j} \text { have the same label } \\ d_{i j}, & \text { otherwise. }\end{cases}
$$

where $0 \leq t \leq 1$ is a decay factor. In this way, the supervised PCA constructs the projection which emphasizes inter-cluster separation.

Supervised PGA for Facial Needle-maps. As mentioned above, the approximation (11) enables us to compute the principal geodesics by applying standard PCA in the tangent plane $T_{\mu} S^{2}$. A straightforward generalization is to replace the standard PCA by the supervised PCA. This has the effect of enhancing the inter-cluster separability of the obtained projection.

Suppose there are $K$ gender-labeled facial needle-maps, and each having $N$ pixel locations. The surface normal at location $l$ in the $k^{t h}$ needle-map is $n_{l}^{k}$. We 
calculate the intrinsic mean $\mu_{l}$ at each pixel location $l$. $n_{l}^{k}$ is then represented by its $\log$ map position $u_{l}^{k}=\log _{\mu_{l}}\left(n_{l}^{k}\right)$ in the tangent plane. $u^{k}=\left[u_{1}^{k}, \ldots, u_{N}^{k}\right]^{T}$ is the log mapped long vector of the $k^{t h}$ training needle-map in the tangent plane $T_{\mu} S^{2}(N)$. The $K$ long vectors form the column-wise data matrix $U=$ $\left[u^{1}|\ldots| u^{K}\right]$. For gender classification, we construct the dissimilarity matrix:

$$
d_{i j}=\left\{\begin{array}{l}
0, \mathrm{i} \text { and } \mathrm{j} \text { are of same gender; } \\
1, \text { otherwise. }
\end{array}\right.
$$

and the corresponding Laplacian matrix is $L$. According to [11, the $p$ leading eigenvectors with the highest eigenvalues of the matrix $U L U^{T}$ form the projection $\Phi$ in the long vector space. Given a facial needle-map, its long vector $u=\left[u_{1}, \ldots, u_{N}\right]^{T}$ can be obtained through log-map of the surface normals, and the corresponding supervised PGA parameters can be calculated as $b=\Phi^{T} u$.

For face images, the dimension $N$ is usually large. The memory storage and manipulation of the $N \times N$ matrix $U L U^{T}$ are usually unfeasible. Since the Laplacian $L$ is an $K \times K$ symmetric positive semi-definite matrix, its eigenvalues are non-negative. Through eigen-decomposition, $L$ can be rewritten as $L=\Phi_{L} \sqrt{\Lambda} \sqrt{\Lambda} \Phi_{L}^{T}=\left(\Phi_{L} \sqrt{\Lambda}\right)\left(\Phi_{L} \sqrt{\Lambda}\right)^{T}$, where $\Phi_{L}$ and $\Lambda$ are the eigenvectors and eigenvalues of $L$. Accordingly,

$$
U L U^{T}=U\left(\Phi_{L} \sqrt{\Lambda}\right)\left(\Phi_{L} \sqrt{\Lambda}\right)^{T} U^{T}=\left(U \Phi_{L} \sqrt{\Lambda}\right)\left(U \Phi_{L} \sqrt{\Lambda}\right)^{T} .
$$

Then, the numerically efficient snap-shot method of Sirovich [13 can be used to compute the eigenvectors and eigenvalues of $U L U^{T}$.

Representing $U L U^{T}$ as $\left(U \Phi_{L} \sqrt{\Lambda}\right)\left(U \Phi_{L} \sqrt{\Lambda}\right)^{T}$, the supervised PGA can be viewed as standard PGA on weighted long vectors. The weight matrices for each data is the component-wise division of $\left(U \Phi_{L} \sqrt{\Lambda}\right)$ and $U$. Some examples of the weight matrices for the ground-truth facial needle-maps are shown in Fig. 2. From the figure, the calculated weight matrices have higher weights in the areas which are used for gender discrimination by human observers. Explained as standard PGA on weighted data, the supervised PGA method is related with the weighted PGA proposed in 12. The difference is the weight matrix adopted in weighted PGA is a common one which can be obtained from a priori knowledge, while in supervised PGA, the weight matrices are different for each data, and they are obtained from the labeled data's pairwise relationships.

\section{Non-Lambertian Shape from Shading}

Faces do not strictly reflect light according to Lambert's law. Therefore, we turn to a new shape-from-shading framework for non-Lambertian facial reflectance developed by Smith and Hancock [7. This method not only imposes a hard non-Lambertian image brightness constraint, but uses a statistical constraint to impose the correct overall shape on the recovered facial needle-maps. 


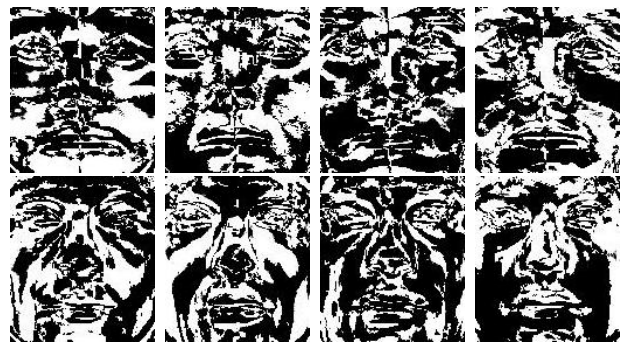

Fig. 2. Examples of the computed weight matrices. The two rows are according to the two directions. The first two columns are females, the last two columns are males.

The brightness constraint uses Torrance and Sparrow reflectance model [8]:

$$
g_{\mathrm{T} \& \mathrm{~S}}\left(\mathbf{N}, \mathbf{L}, \mathbf{V},\left\{\rho_{d}, \rho_{s}, \nu, L\right\}\right)=L \rho_{d} \mathbf{N} \cdot \mathbf{S}+\frac{L \rho_{s} e^{-\nu^{2} \arccos \left(\mathbf{N} \cdot \frac{\mathbf{L}+\mathbf{V}}{\pi \mathbf{L}+\mathbf{V}}\right)^{2}}}{\mathbf{N} \cdot \mathbf{V}},
$$

where $\rho_{s}$ is the specular coefficient, $\nu$ the surface roughness, $L$ the light source intensity and, $\rho_{d}$ is the diffuse albedo. The brightness error can be defined as a function of $n \in S^{2}: f(n)=\left(g\left(\Phi(n), \mathbf{L}, \mathbf{V},\left\{\rho_{d}, \rho_{s}, \nu, L\right\}\right)-I\right)^{2}$, where $\Phi$ : $S^{2} \rightarrow R^{3}$ is an embedding. Making use of the exponential map, the local gradient of $f$ can be approximated in terms of a vector on the tangent plane $T_{n} S^{2}$ :

$$
\nabla f(n)=\left(\frac{f\left(\operatorname{Exp}_{n}\left[(\epsilon, 0)^{T}\right]\right)-f(n)}{\epsilon}, \frac{f\left(\operatorname{Exp}_{n}\left[(0, \epsilon)^{T}\right]\right)-f(n)}{\epsilon}\right)^{T} .
$$

Gradient descent is used to locate the local minimum of the brightness error function: $n_{t+1}=\operatorname{Exp}_{n_{t}}\left(-\gamma \nabla f\left(n_{t}\right)\right)$, where $\gamma$ is the step size.

The new SFS method also makes use of a surface height model constructed by applying PCA to a set of facial surface samples. Suppose $\Psi_{i}$ is the $i^{\text {th }}$ principal eigenvector of samples' covariance matrix. The surface normals satisfying the statistical model can be expressed in terms of the parameter vector $b$ :

$$
\mathbf{n}_{\mathbf{b}}(x, y)=\left(\sum_{i=1}^{m} b_{i} \partial_{x} \Psi_{i}(x, y), \sum_{i=1}^{m} b_{i} \partial_{y} \Psi_{i}(x, y), 1\right)^{T} .
$$

In our experiments, we choose $m$ to be 50 to maintain shape recovery accuracy while reducing noise. In order to apply this statistical constraint to $n(x, y)$ satisfying brightness constraint, the parameter vector $\mathbf{b}^{*}$ must be located minimizing the distance between $n(x, y)$ and $n_{b^{*}}(x, y)$. In [7], this minimization task is poses as that of minimizing the squared error between the surface gradients of $n(x, y)$ and $n_{b^{*}}(x, y)$. The optimal solution is: $\mathbf{b}^{*}=\left(\Psi^{T} \boldsymbol{\Psi}\right)^{-1} \Psi^{T} \mathbf{G}$, where $G$ are the surface gradients of $n(x, y)$, and $\Psi$ is the surface gradient of the eigenvectors.

A detailed description of this non-Lambertian SFS method is given in [7]. 


\section{Classification}

With a set of gender-labeled facial needle-maps, we obtain the projection matrix using supervised PGA. Both training and test needle-maps are represented by supervised PGA parameters. A Bayes classifier is used for gender classification.

Let $C_{f}$ and $C_{m}$ denote the female and male gender classes, $x$ denote the p dimensional supervised PGA parameters of a test facial needle-map. Then according to the Bayes law, the probability that $x$ is of class $C_{i}$ is:

$$
P\left(C_{i} \mid x\right)=\frac{P\left(x \mid C_{i}\right) P\left(C_{i}\right)}{\sum_{i \in\{f, m\}} P\left(x \mid C_{i}\right)} .
$$

We assume $x$ follow a Gaussian distribution for the different genders, and that the parameter vector mean and covariance of class $C_{i}$ are $\mu_{i}$ and $\Sigma_{i}$. Then,

$$
P\left(x \mid C_{i}\right)=\frac{1}{\sqrt{(2 \pi)^{p} \operatorname{det}\left(\Sigma_{i}\right)}} \exp \left[-\frac{1}{2}\left(x-\mu_{i}\right)^{T} \Sigma_{i}^{-1}\left(x-\mu_{i}\right)\right] .
$$

The a posteriori probability $P\left(C_{f} \mid x\right)$ and $P\left(C_{m} \mid x\right)$ are computed by applying (44) and substituting equal a priori probability into (3). If $P\left(C_{f} \mid x\right)>P\left(C_{m} \mid x\right)$, then the face is classified as female. Otherwise, the face is classified as male.

\section{Experimental Results}

In this section, we report experiments on both ground-truth face needle-maps and needle-maps recovered from images using SFS. The ground-truth data are from the Max-Planck Face Database [14], 15]. It comprises 200 normalized 3D range images of human heads (100 females and 100 males), which are used to construct the statistical model for non-Lambertian SFS. The 168 (94 females and 74 males) brightness images with neutral expression and no glasses are from the AR Face Database 16. These images are aligned using the centers of the eyes, the tip of the nose, and the middle of the mouth.

\subsection{Experiments on Ground-Truth Data}

We first evaluate the performance of supervised PGA by examining the gender discriminating powers of the eigenvectors. Then, we compare classification results using supervised PGA parameters with those using PGA parameters.

Discriminating Powers are calculated using the criterion function introduced in [17]: $J(\xi)=\operatorname{tr}\left(S_{w}^{-1} S_{b}\right)=\sum_{k=1}^{d} \lambda_{k}$. The discriminating power of each of the 10 leading supervised PGA eigenvectors are shown in Fig. 3, compared with those using standard PGA. From the figure, it is clear that there is greater discriminating power in the first supervised PGA eigenvector. By comparison, the discriminating power of standard PGA eigenvectors are more widely distributed. This confirms our assumption that by making use of the interclass structure, supervised PGA improves the gender discriminating power of the leading principal geodesics. 

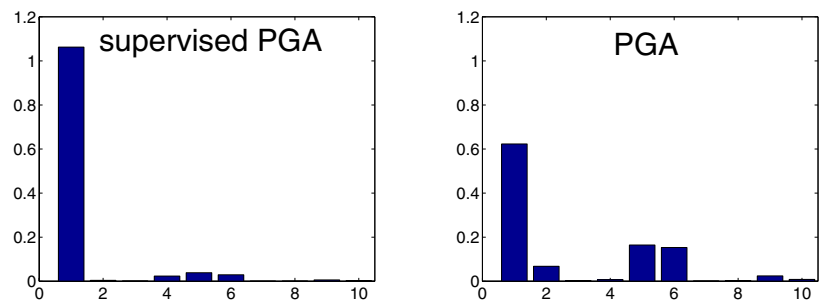

Fig. 3. Discriminating powers of the 10 leading eigenvectors

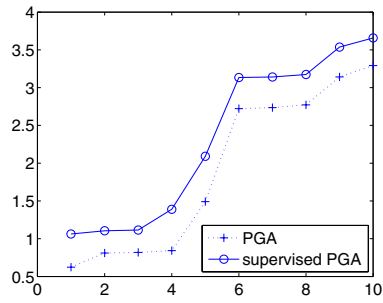

Fig. 4. Cumulative discriminating powers

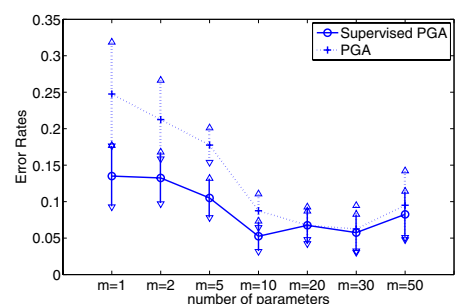

Fig. 5. Classification error rates for ground-truth data

Fig. 4 shows the cumulative gender discriminating powers. From the figure, it is clear that supervised PGA has significantly larger cumulative discriminating powers than standard PGA in the first 10 dimensions. This offers the expectation of higher classification accuracy using leading supervised PGA parameters.

Classification Results. 160 needle-maps are randomly selected from the 200 for use as training data to obtain the projection matrix, the remaining 40 as test data. Gender classification is performed by applying the Bayes classifier to the leading $p(p=1,2,5,10,20,30,50)$ parameters for both supervised PGA and standard PGA. We repeat the randomization 10 times for each $p$. The average error rates and variance are shown in Figure 5, which exhibits interesting effects. First, when $p \leq 10$, using supervised PGA achieves significantly better accuracies than using standard PGA. This is expected from the cumulative discriminating powers. Second, the lowest error rate is $5.25 \%$ and $6.25 \%$ for the two methods, which indicate the feasibility of accurate gender classification based on facial shape information revealed by facial needle-maps. Finally, for both methods, the classification accuracy has an inflexion with increasing of $p$. It is consistent with the 'curse of dimensionality' phenomenon, as well as human classification which is based on just a few important attributes [17]. The redundant or irrelevant information in higher dimensions causes deteriorate of the classification accuracy. 


\subsection{Experiments with Brightness Images}

Results of SFS. Four recovered needle-maps and surfaces are shown in Fig. 6. It is clear that the recovered needle-maps and the surfaces give realistic shape, overcoming the well-known local convexity-concavity instability problem.

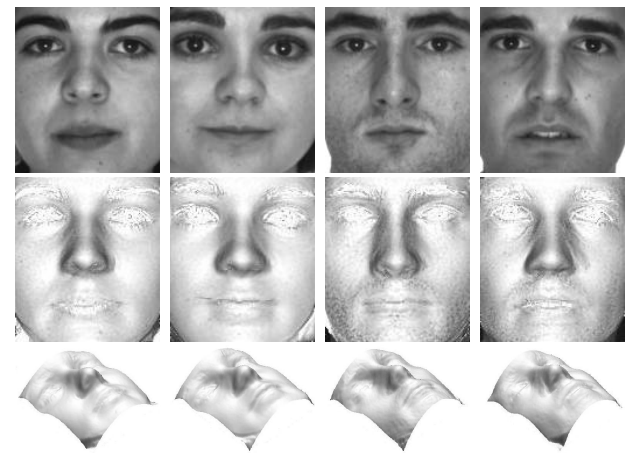

Fig. 6. Recovered needle-maps. First two columns are females, last two are males. From top to bottom are the input images, the recovered needle-maps, the recovered surfaces.

Discriminating Powers. We repeat the analysis of the discriminating powers of the 10 leading eigenvectors and the cumulative discriminating powers, and plot them in Figs. 7 and 8. From Fig. 7, it is clear that the first supervised PGA eigenvector encodes significantly more gender discriminating power. It is consistent with those of ground-truth data. However, the cumulative discriminating powers in Fig. 8 exhibit some systematic differences. The discriminating power of supervised PGA is superior to that of standard PGA when using only the first parameter. As the number of parameters increases, there is no significant difference between the two methods.
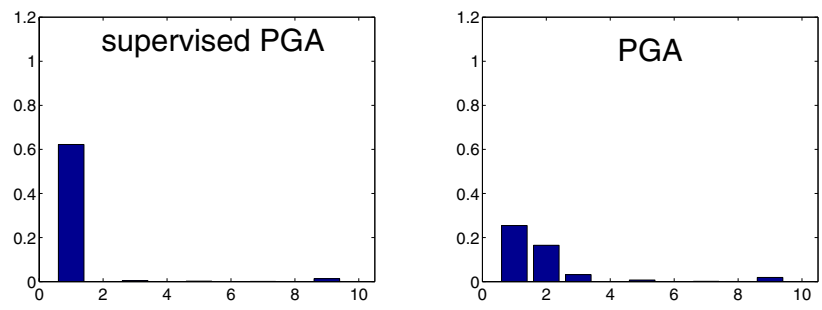

Fig. 7. Discriminating powers of the 10 leading eigenvectors

Classification Results. We randomly select 134 from the 168 brightness images as training data, and the remaining 34 as test data. The procedure described above is repeated. The classification results are shown in Figure 9. As expected 


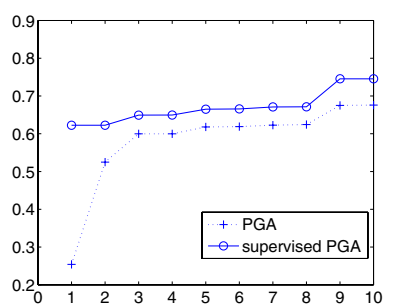

Fig. 8. Cumulative discriminating powers

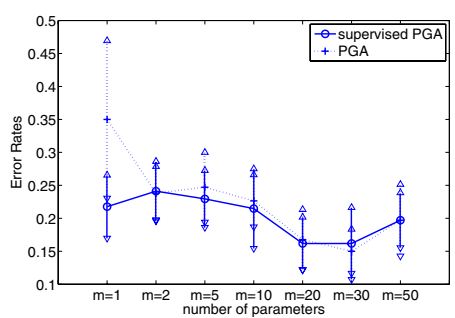

Fig. 9. Classification error rates for the recovered needle-maps

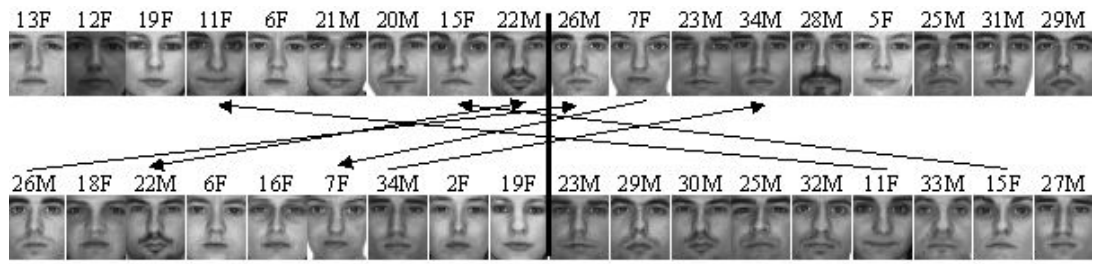

Fig. 10. Visualization of the classification

from the cumulative discriminating power, the classification results using supervised PGA are only superior to using standard PGA when $p=1$. With increasing $p$, the advantage is not obvious. The best classification accuracy is about $84 \%$ for both methods, which confirms that the recovered facial needle-maps convey gender information. As the results on ground-truth data, the results on recovered needle-maps also confirm the 'curse of dimensionality' phenomenon.

For one classification, we arrange the test images into series according to their a posteriori classification probabilities. The middle portion of the series corresponding to gender overlap are shown in Fig. 10. The first row is using supervised PGA, and the second row is using standard PGA. The line in the middle is the Bayes decision boundary between the two genders (females to the left and males to the right). Each image is labeled with its index and actual gender. From the figure we can see, using supervised PGA, the misclassified faces $(21 \mathrm{M}, 20 \mathrm{M}, 22 \mathrm{M}, 7 \mathrm{~F}, 5 \mathrm{~F})$ are more concentrated in the overlap area, while in the case of standard PGA, the misclassified $(26 \mathrm{M}, 22 \mathrm{M}, 34 \mathrm{M}, 11 \mathrm{~F}, 15 \mathrm{~F})$ are more dispersed. Faces $26 \mathrm{M}, 34 \mathrm{M}, 11 \mathrm{~F}$ and $15 \mathrm{~F}$ which are misclassified using standard PGA, are correctly classified using supervised PGA.

\section{Conclusion}

In this paper, we perform gender classification on 2.5D facial surface normals. A recently reported non-Lambertian SFS method is used to recover facial needlemaps from brightness images. And a novel supervised PGA technique is 
developed to parameterize needle-maps so as to maximize gender discrimination. Experimental results reveal that supervised PGA improves gender discriminating powers of the leading eigenvectors. The high classification accuracy supports the feasibility of gender discrimination based on facial needle-maps.

Supervised PGA only appears to improve the classification on the recovered needle-maps using the first dimension of parameters. Establishing the reason for this is our first future work. Another topic is to investigate the distribution of the weight matrices. This may have some potential for unsupervised learning.

\section{References}

1. Buchala, S., Davey, N., Gale, T., Frank, R.: Principal component analysis of gender, ethnicity, age, and identity of face images. In: IEEE ICMI 2005 (2005)

2. Lu, X., Chen, H., Jain, A.: Multimodal facial gender and ethnicity identification. In: ICB 2006, pp. 554-561 (2006)

3. Moghaddam, B., Yang, M.: Learning gender with support faces. IEEE Trans. on PAMI 24(5), 707-711 (2002)

4. Bruce, V., Burton, A., Hanna, E., Healey, P., Mason, O., Coombes, A., Fright, R., Linney, A.: Sex discrimination: how do we tell the difference between male and female faces? Perception 22, 131-152 (1993)

5. Burton, A., Bruce, V., Dench, N.: What's the difference between men and women? evidence from facial measurement. Perception 22, 153-176 (1993)

6. Wu, J., Smith, W., Hancock, E.: Gender classification using shape from shading. In: BMVC 2007, pp. 499-508 (2007)

7. Smith, W., Hancock, E.: A new framework for grayscale and colour non-lambertian shape-from-shading. In: ACCV 2007, pp. 869-880 (2007)

8. Torrance, K., Sparrow, E.: Theory for off-specular reflection from roughened surfaces. Journal of the Optical Society of America 57, 1105-1114 (1967)

9. Fletcher, P., Joshi, S., Lu, C., Pizer, S.: Principal geodesic analysis for the study of nonlinear statistics of shape. IEEE Trans. on Medical Imaging 23, 995-1005 (2004)

10. Pennec, X.: Probabilities and statistics on riemannian manifolds: A geometric approach. Technical Report RR-5093, INRIA (2004)

11. Koren, Y., Carmel, L.: Robust linear dimensionality reduction. IEEE Trans. on VCG 10(4) (2004)

12. Wu, J., Smith, W., Hancock, E.: Weighted principal geodesic analysis for facial gender classification. In: Rueda, L., Mery, D., Kittler, J. (eds.) CIARP 2007. LNCS, vol. 4756, pp. 331-339. Springer, Heidelberg (2007)

13. Sirovich, L.: Turbulence and the dynamics of coherent structures. Quart. Applied Mathematics XLV(3), 561-590 (1987)

14. Troje, N., Bulthoff, H.: Face recognition under varying poses: The role of texture and shape. Vision Research 36, 1761-1771 (1996)

15. Blanz, V., Vetter, T.: A morphable model for the synthesis of 3d faces. In: SIGGRAPH 1999 Conference Proceedings, pp. 187-194 (1999)

16. Martinez, A., Benavente, R.: The ar face database. CVC Technical Report (1998)

17. Devijver, P., Kittler, J.: Pattern Recognition: A Statistical Approach. PrenticeHall (1982) 